\title{
Pyrethroid Resistance in Anopheles gambiae Not Associated with Insecticide-Treated Mosquito Net Effectiveness Across Sub-Saharan Africa
}

\author{
David A. Larsen* and Rachael L. Church \\ Syracuse University Department of Public Health, Syracuse, New York
}

\begin{abstract}
Pyrethroid resistance is a major concern for malaria vector control programs that predominantly rely on insecticide-treated mosquito nets (ITNs). Contradictory results of the impact of resistance have been observed during field studies. We combined continent-wide estimates of pyrethroid resistance in Anopheles gambiae from 2006 to 2017 , with continent-wide survey data to assess the effect of increasing pyrethroid resistance on the effectiveness of ITNs to prevent malaria infections in sub-Saharan Africa. We used a pooled-data approach and a meta-regression of survey regions to assess how pyrethroid resistance affects the association between ITN ownership and malaria outcomes for children 6 to 59 months of age. ITN ownership reduced the risk of malaria outcomes according to both the pooled and metaregression approaches. According to the pooled analysis, there was no observed interaction between ITN ownership and estimated level of pyrethroid resistance (likelihood ratio [LR] test, 1.127 for malaria infection confirmed by the rapid diagnostic test, $P=0.2885$; LR test $=0.161$ for microscopy-confirmed malaria infection, $P=0.161$; LR test $=0.646$ for moderate or severe anemia, $P=0.4215)$. Using the meta-regression approach to determine the level of pyrethroid resistance did not explain any of the variance in subnational estimates of ITN effectiveness for any of the outcomes. ITNs decreased the risk of malaria independent of the levels of pyrethroid resistance in malaria vector populations.
\end{abstract}

\section{INTRODUCTION}

Insecticide-treated mosquito nets (ITNs) have been one of the most effective public health interventions of the 21 st century and prevent millions of deaths caused by the malaria parasite. ${ }^{1}$ Before their widescale deployment, more than 1 million people died annually across sub-Saharan Africa from malaria infections, ${ }^{2}$ particularly the Plasmodium falciparum parasite, which caused an additional 500 million cases. Broad scaleup of ITNs across the continent in the early and mid-2000s ${ }^{3}$ contributed to this annual burden being cut in half by $2015 .{ }^{4}$ The 2018 World Malaria Report found that all species of malaria were responsible for 435,000 global deaths and 219 million global cases in $2017,{ }^{5}$ which was a huge reduction from estimates earlier during the century. However, it is worrisome that the 2018 World Malaria Report observed the first annual increase in global malaria cases since $2005 .^{5}$

Mosquito nets provide a personal barrier to prevent infective bites from malaria vectors that predominantly bite at night. ${ }^{6}$ An untreated mosquito net will protect the person sleeping beneath from an infective bite of an infected mosquito. However, that infective malaria vector will likely seek a blood meal elsewhere, thus continuing malaria transmission. When the mosquito net is treated with insecticide and the mosquito is susceptible, contact with the insecticide kills the mosquito and ends that mosquito's capacity to continue transmitting malaria. ${ }^{7}$ The subsequent effect of ITNs on malaria transmission is profound. ${ }^{8}$ When a high number of ITNs are used within a community, a mass killing effect caused by the insecticide provides community-level protection, sufficiently affecting the malaria vector populations so that people who do not have an ITN are protected in similar ways as those who do have an ITN. ${ }^{9,10}$

The current malaria control strategy relies on pyrethroids, ${ }^{11}$ although combination nets that incorporate synergistic catalysts such as piperonyl butoxide and chlorfenapyr that make

*Address correspondence to David A. Larsen, Syracuse University Department of Public Health, 430C White Hall Ave., Syracuse, NY 13244. E-mail: dalarsen@syr.edu the pyrethroids more deadly are now available. ${ }^{12-14}$ Pyrethroids target the central nervous system to kill mosquitoes and reduce the transmission of malaria by incapacitating the vector. ${ }^{15}$ Fears about malaria vectors developing pyrethroid resistance were raised at the inception of ITNs, ${ }^{15}$ and early studies demonstrated widespread resistance. ${ }^{16,17}$ Although alarming, it remains to be seen just how pyrethroid resistance will affect malaria control and malaria trends. ${ }^{18-20}$

Linking pyrethroid resistance in malaria vectors to ITN effectiveness to prevent malaria infections and reduce malaria transmission has been more challenging than documenting the spread of resistance. Some studies have suggested that in areas with pyrethroid resistance, ITNs are less effective. ${ }^{21,22}$ Others have found that ITNs are still associated with a reduced risk of malaria despite high levels of pyrethroid resistance. ${ }^{23-26}$ These studies generally struggle with four separate issues. First, because of the inability to randomly allocate ITN access, these studies have selection bias; households with ITN access are more likely to be predisposed to a lower risk of malaria transmission than households without ITN access, independent of the effect of ITNs. ${ }^{27}$ Second, some of these studies have not included estimates of ITN effectiveness, and the studies that have included a measure of ITN effectiveness used ITN use as their measure of effectiveness. ITN use may not be the best measure for understanding the impact of pyrethroid resistance on ITN effectiveness. Even if malaria vectors are resistant to the insecticide in the ITN, the mosquito netting still provides a physical barrier, and consistent use will decrease mosquito exposure and, thus, malaria risk. ${ }^{28}$ This is evidenced by ITNs with holes having reduced effectiveness to prevent malaria in areas of pyrethroid resistance. ${ }^{29}$ Third, each of the studies was confined to a particular study site. Investigating phenomena at particular study sites limits the variability of resistance available because the prevalence of broad-spectrum resistance is not incorporated in the study. Fourth, the studies used an overly simplistic assessment of insecticide resistance, typically with dichotomized lowresistance and high-resistance areas that prevented the examination of a dose-response relationship between ITN effectiveness and pyrethroid resistance. 
We attempted to address these four challenges and gain a better understanding of how widespread pyrethroid resistance is affecting the ability of ITNs to reduce malaria transmission. We used nationally representative surveys across sub-Saharan Africa and recently published nearly continentwide estimates of pyrethroid resistance of Anopheles gambiae in sub-Saharan Africa ${ }^{30}$ to address the issues of geographic limitation, a limited resistance spectrum, and oversimplification of the statistical measure of insecticide resistance. We further used exact matching to minimize the influence of selection bias and a measure of ITN ownership rather than ITN use to reduce the residual confounding of the effect of mosquito netting as a barrier.

\section{METHODS}

Search strategy and selection criteria. We used data from nationally representative demographic and health surveys (DHS) to assess the relationship between insecticide resistance and the effectiveness of ITNs. All DHS conducted in sub-Saharan Africa between 2006 and 2015 that were publicly available as of January 15, 2020, were considered for inclusion in the analyses if the survey contained information regarding an outcome (malaria infection status for children, either measured using a rapid diagnostic test or measured using microscopy, or anemia status measured using a hemocue rapid hemoglobin assessment) and if the survey contained information regarding household ITN ownership. We further limited these surveys to those conducted in areas with an estimated prevalence of pyrethroid resistance. ${ }^{30}$ The DHS program is funded by the United States Agency for International Development and assists lower-income countries with conducting nationally representative surveys primarily aimed at measuring trends in child mortality and women's fertility with statistical power at the regional (sub-national) level. Additionally, these surveys gather a host of information regarding numerous factors associated with health, including malaria indicators when the survey is conducted in a malariaendemic country.

Outcome measures. We considered three separate outcome measures for children 7 to 49 months of age: confirmation of malaria infection using a rapid diagnostic test; confirmation of malaria infection using microscopy; or hemoglobin levels $<10 \mathrm{~g} / \mathrm{dL}$ (moderate to severe anemia). Anemia is an historical indicator of malaria transmission that is known to be associated with effective malaria control such as ITNs. ${ }^{31}$

ITN ownership measure. We defined ITN ownership as a household that owned at least one ITN and had at least one child living in that household.

Pyrethroid resistance measure. We extracted modeled estimates of pyrethroid resistance among the An. gambiae complex ${ }^{30}$ to the year and cluster geocoordinates for each survey included in the study using the Raster package ${ }^{32}$ in $R$ version 3.6.2. ${ }^{33}$ In brief, Hancock et al. ${ }^{30}$ estimated pyrethroid resistance at the population scale for eastern and western Africa using a global dataset of CDC bottle bioassays of four pyrethroid types (deltamethrin, permethrin, lambda-cyhalothrin, and cypermethrin). ${ }^{34}$

Exact matching of observations. Selection bias presents the largest challenge to validity when assessing ITN effectiveness during observational studies. We followed an exact matching approach similar to that used for the analysis of
ITN effectiveness conducted by Lim et al. to minimize this selection bias ${ }^{35}$; we matched households based on the probability of owning an ITN. Specifically, we matched households within survey datasets based on the following covariate pattern: region of the country as per the DHS, wealth categorized as rich or poor, mother's education categorized as any or none, and the house categorized as being in an urban or rural location. Matching was performed using the Matchlt package $^{36,37}$ in $R$ version 3.6.2. ${ }^{33}$

Statistical analysis. We used two separate approaches to assess the influence of pyrethroid resistance on the effectiveness of ITNs.

Pooled analysis approach. We combined all survey data that fulfilled the inclusion criteria and that exactly matched into a single dataset. We extracted the estimates of resistance, seasonality of malaria transmission from the Mapping Malaria Risk in Africa (MARA) maps, ${ }^{38}$ estimates of the Plasmodium falciparum parasite prevalence rate (PfPR) for children 2 to 10 years from the Malaria Atlas Project, ${ }^{39}$ and the relative abundance of the Anopheles gambiae vector ${ }^{40}$ to the cluster geocoordinates using the Raster package ${ }^{32}$ in $\mathrm{R}$ version 3.6.2. ${ }^{33}$ The PfPR estimates were only available for the years 2000 to 2015, from the MalariaAtlas package; therefore, we limited the analysis to surveys conducted during that time period.

We first estimated the association between ITN ownership and malaria outcomes without including estimates of resistance using a logistic regression approach with the matched group as a random intercept (Equation 1). Then, we estimated the relationship between ITN effectiveness and the prevalence of resistance by comparing log likelihoods of a saturated model (Equation 2) with those of a full model (Equation 3). We adjusted all analyses for child's age categorized as years (younger than 1 year, 1 year, 2 years, 3 years, 4 years), household wealth quintile, mother's education (none, some primary, completed primary or higher), whether the data were collected during the transmission season, household location (urban or rural), and PfPR levels (continuous). The three separate outcomes were modeled in the same manner, with one for the outcome of malaria infection confirmed by a rapid diagnostic test and the other for the outcome of microscopy-confirmed malaria infection. We conducted an analysis of all observations in the dataset; then, we limited the analysis to those observations for which the relative abundance of An. gambiae was $>0.5$. Analyses were conducted using Stata version 15.1.

Equation (1): Representation of the pooled analysis of ITN effectiveness when excluding resistance estimates wherein $\pi_{\mathrm{ijklm}}$ is a dichotomous outcome for child $\mathrm{i}$ in household $\mathrm{j}$ in cluster $\mathrm{k}$ in survey $\mathrm{I}$ in matched group $\mathrm{m}, \mathrm{ITN}_{\mathrm{j}}$ represents whether the household has access to any ITN, $\mathrm{C}_{\mathrm{ijk}}$ is a vector of child characteristics, $\mathrm{H}_{\mathrm{j}}$ is a vector of household characteristics, $S_{k}$ is a vector of cluster characteristics, and $\zeta_{m}$ is a random intercept for matched group $m$ that is assumed to be normally distributed with a mean of zero.

$$
\begin{aligned}
y_{i j k l m} \mid \pi_{i j k l m} & \sim \operatorname{Binomial}\left(1, \pi_{i j k l m}\right) \\
\operatorname{logit}\left(\pi_{i j k l m}\right)=B_{1} I T N_{j} \times & \operatorname{Res}_{k}+B_{2} I T N_{j}+\chi C_{i j k}+\delta H_{j}+\kappa S_{k} \\
\zeta_{m} & \sim N(0, \varphi)
\end{aligned}
$$

Equation (2) (saturated model): Representation of the pooled analysis of ITN effectiveness when interacting 


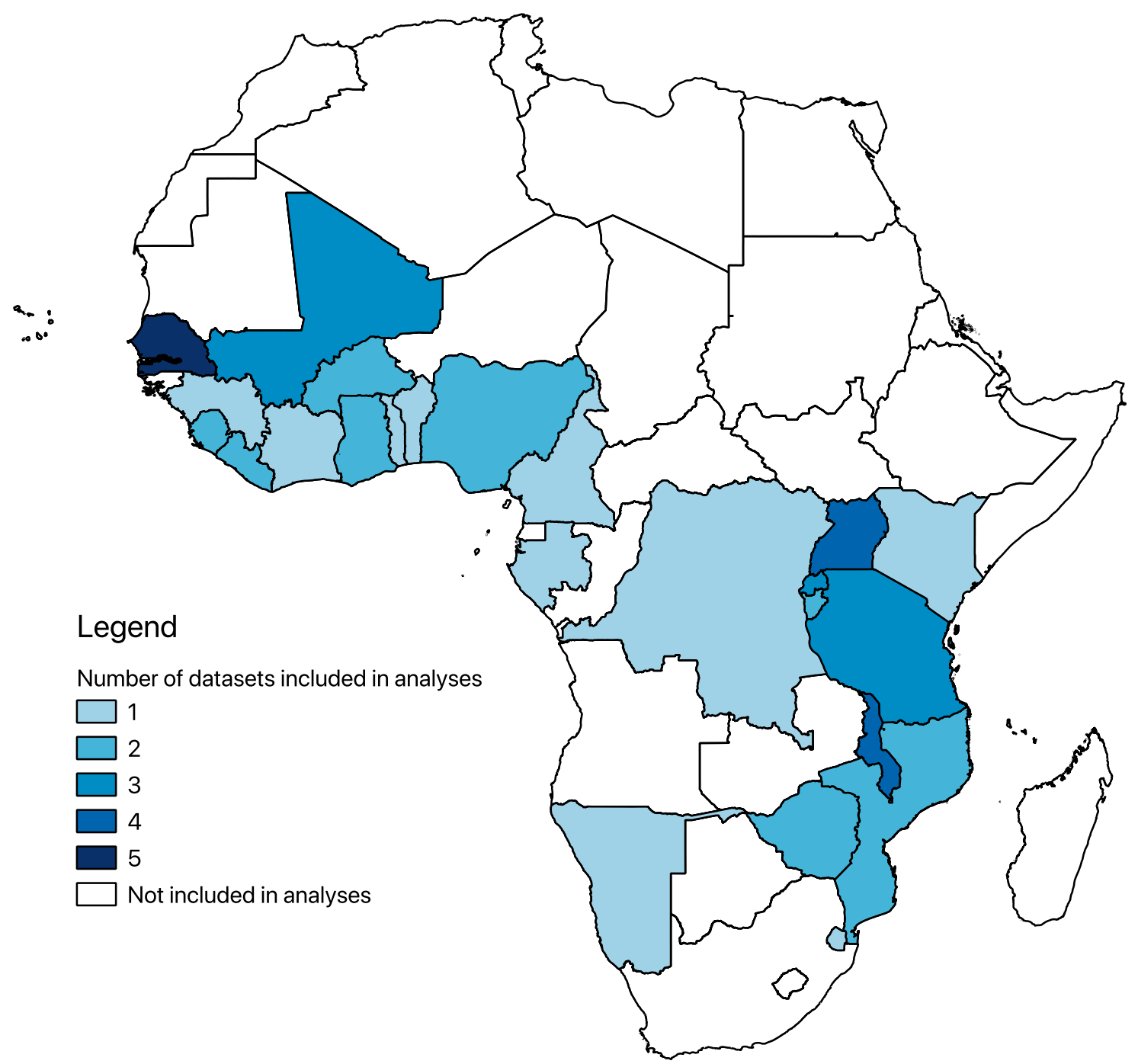

FIGURE 1. Geographic distribution of data included in the analyses. This figure appears at www.ajtmh.org.

resistance estimates with ITN ownership wherein $\pi_{i j k l m}$ is a dichotomous outcome for child $\mathrm{i}$ in household $\mathrm{j}$ in cluster $\mathrm{k}$ in survey I in matched group $\mathrm{m}$, ITN $\mathrm{N}_{\mathrm{j}}$ represents whether the household has access to any ITN, Res $\mathrm{R}_{\mathrm{k}}$ is the level of pyrethroid resistance access in the community, $\mathrm{C}_{\mathrm{ijk}}$ is a vector of child characteristics, $\mathrm{H}_{\mathrm{j}}$ is a vector of household characteristics, $S_{k}$ is a vector of cluster characteristics, and $\zeta_{\mathrm{m}}$ is a random intercept for matched group $m$ that is assumed to be normally distributed with a mean of zero.

$$
\begin{aligned}
y_{i j k l m} \mid \pi_{i j k l m} & \sim \operatorname{Binomial}\left(1, \pi_{i j k l m}\right) \\
\operatorname{logit}\left(\pi_{i j k l m}\right)= & B_{1} / T N_{j} \times \operatorname{Res}_{k}+B_{2} / T N_{j} \\
& +B_{3} \operatorname{Res}_{k}+\chi C_{i j k}+\delta H_{j}+\kappa S_{k} \\
& \zeta_{m} \sim N(0, \varphi)
\end{aligned}
$$

Equation (3) (full model): Representation of the pooled analysis of ITN effectiveness when including resistance estimates and ITN ownership but no interaction wherein $\pi_{\mathrm{ijklm}}$ is a dichotomous outcome for child $i$ in household $\mathrm{j}$ in cluster $\mathrm{k}$ in survey I in matched group $\mathrm{m}$, ITN $\mathrm{N}_{\mathrm{j}}$ represents whether the household has access to any ITN, Res ${ }_{k}$ is the level of pyrethroid resistance access in the community, $\mathrm{C}_{\mathrm{ijk}}$ is a vector of child

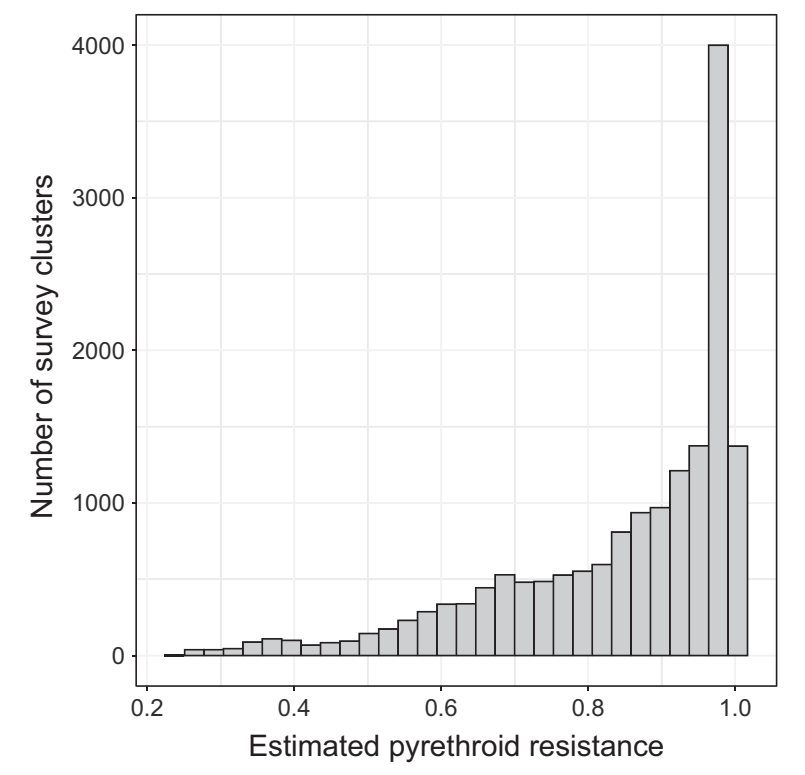

FIGURE 2. Distribution of estimated pyrethroid resistance across datasets included in the analyses. 
TABLE 1

Association between ITN ownership and various outcomes without considering pyrethroid resistance

\begin{tabular}{|c|c|c|c|}
\hline Outcome & Adjusted odds ratio $(95 \% \mathrm{Cl})$ of ITN ownership & $P$ value & No. of children (matched groups) \\
\hline RDT positivity & $0.91(0.88-0.95)$ & $<0.001$ & $103,572(3,236)$ \\
\hline Microscopy positivity & $0.90(0.86-0.95)$ & $<0.001$ & $92,718(2,869)$ \\
\hline Moderate or severe anemia & $0.95(0.93-0.98)$ & $<0.001$ & $131,679(4,294)$ \\
\hline
\end{tabular}

ITN = insecticide-treated mosquito net; RDT = rapid diagnostic test. Children were matched based on the probability of ITN ownership. Models were adjusted for urban/rural location, child's age, wealth quintile, mother's education, Plasmodium falciparum parasite prevalence rate (PfPR), malaria transmission season, and survey dataset.

characteristics, $\mathrm{H}_{\mathrm{j}}$ is a vector of household characteristics, $\mathrm{S}_{\mathrm{k}}$ is a vector of cluster characteristics, and $\zeta_{\mathrm{m}}$ is a random intercept for matched group $m$ that is assumed to be normally distributed with a mean of zero.

$$
\begin{gathered}
y_{i j k l m} \mid \pi_{i j k l m} \sim \operatorname{Binomial}\left(1, \pi_{i j k l m}\right) \\
\operatorname{logit}\left(\pi_{i j k l m}\right)=B_{1} I T N_{j}+B_{2} \operatorname{Res}_{k}+\chi C_{i j k}+\delta H_{j}+\kappa S_{k} \\
\zeta_{m} \sim N(0, \varphi)
\end{gathered}
$$

Meta-regression approach. We parsed all survey data meeting the inclusion criteria to the DHS region level. We extracted the estimates of pyrethroid resistance, the PfPR for children 2 to 10 years of age from the Malaria Atlas Project, ${ }^{39}$ and the relative abundance of the Anopheles gambiae vector $^{40}$ to the DHS region level using the Raster package ${ }^{32}$ in $\mathrm{R}$ version 3.6.2. ${ }^{33}$

Then, we estimated the effectiveness of ITNs for each DHS region as described previously using Equation (1). We conducted a meta-regression of ITN effectiveness within DHS regions using the metafor package ${ }^{41}$ in $\mathrm{R}$ version 3.6.2, ${ }^{33}$ with the prevalence of pyrethroid resistance at the region level serving as the predictor, a priori hypothesizing that a randomeffects meta-analysis model would be most appropriate. We also tested for interactions between pyrethroid resistance and both PfPR, as well as the abundance of Anopheles gambiae.

\section{RESULTS}

Data available. We identified 77 survey datasets conducted in sub-Saharan Africa between 2006 and 2015, that were publicly available as of January 15, 2020, with information regarding ITN ownership, malaria infection in children, or the anemia status of children. Of these 78 surveys, 28 were excluded from any analysis because of the following reasons: 12 surveys contained no information about malaria infection or anemia status; three surveys contained no information about household ITN ownership; and seven surveys could not be matched to resistance estimates (the surveys were conducted in Angola, Sao Tome and Principe, or Madagascar, where no resistance estimates were available).
Another six surveys had no cluster geocoordinates; therefore, they were excluded from the pooled analysis but included in the meta-regression approach. Figure 1 shows the geographical distribution of 55 surveys included in the analyses.

Variation of pyrethroid resistance. Modeled estimates of An. gambiae complex pyrethroid resistance with survey clusters ranged from 23.3\% to 99.9\% (median level, 90.4\%) (Figure 2). Pyrethroid resistance was associated with decreased levels of modeled PfPR within survey clusters (rho $=-0.3365 ; N=15,484$ clusters) and decreased relative abundance of An. gambiae (rho $=-0.2560 ; N=$ 16,051 clusters).

Effect of pyrethroid resistance on the association between ITNs and malaria outcomes: pooled analysis approach. After exact matching and adjusting for known predictors but without accounting for resistance, ownership of an ITN was associated with a reduction in the risk of a malaria infection as well as having moderate or severe anemia (Table 1). After accounting for resistance, there was no evidence of an interaction between ITN ownership and pyrethroid resistance levels for any of the outcomes (Table 2). ITN ownership was still associated with a reduction in the risk of malaria infection as well as having moderate or severe anemia. Limiting the analysis to areas with $>50 \%$ An. gambiae did not change the results.

Effect of pyrethroid resistance on the association between ITNs and malaria outcomes: meta-regression approach. Within-DHS region-specific estimates of the effectiveness of ITNs were available for 349 regions for the outcome of malaria infection confirmed by a rapid diagnostic test, for 243 regions for the outcome of microscopy-confirmed malaria infection, and for 442 regions for the outcome of moderate or severe anemia. According to unadjusted meta-analyses, ITN ownership was associated with a reduced risk of malaria infection confirmed by a rapid diagnostic test and microscopyconfirmed malaria infection, but not moderate or severe anemia (Table 3). Pyrethroid resistance did not explain any of the regional variance in the effectiveness of ITN ownership against the outcomes (Figure 3), nor did any interaction between pyrethroid resistance and either the PfPR or the abundance of An. gambiae.

TABLE 2

Likelihood ratio test including an interaction term for pyrethroid resistance and ITN ownership (comparing the model from Equation 2 to the model from Equation 3)

\begin{tabular}{lcr}
\hline \multicolumn{1}{c}{ Outcome } & $\begin{array}{c}\text { Likelihood ratio test for } \\
\text { interaction between } \\
\text { pyrethroid resistance and } \\
\text { ITN ownership }\end{array}$ & $\begin{array}{c}\text { No. of children } \\
\text { (matched groups) }\end{array}$ \\
\hline RDT positivity & 0.013 & 0.9937 \\
Microscopy positivity & 0.148 & 0.9288 \\
Moderate or severe anemia & 0.012 & 0.9940 \\
\hline ITN = insecticide-treated mosquito net; RDT = rapid diagnostic test. Children were matched based on the probability of ITN ownership. Models were adjusted for urban/rural location, child's age,
\end{tabular}

wealth quintile, mother's education, Plasmodium falciparum parasite prevalence rate (PfPR), malaria transmission season, and survey dataset. 
TABLE 3

Results of the unadjusted meta-analyses of the effect of ITN ownership on outcomes

\begin{tabular}{lcrr}
\hline \multicolumn{1}{c}{ Outcome } & Odds ratio $(95 \% \mathrm{Cl})$ & $P$ value & $I^{2}$ \\
\hline RDT positivity & $0.912(0.847-0.982)$ & 0.0193 & $0.00 \%$ \\
Microscopy positivity & $0.878(0.803-0.959)$ & 0.0040 & 247 \\
Moderate or severe anemia & $0.970(0.920-1.022)$ & 0.2518 & 165 \\
\hline DHS = demographic and health surveys; ITN = insecticide-treated mosquitonet; RDT = rapid diagnostic test. DHS datawere parsed based on region. Children were matched based onthe probability
\end{tabular}
of ITN ownership. Region-specific models were adjusted for urban/rural location, child's age, wealth quintile, and mother's education (as shown in Equation 1).

\section{DISCUSSION}

These results suggest that the effectiveness of ITNs is not affected by the prevalence of pyrethroid resistance across sub-Saharan Africa. ITN ownership was associated with a reduced risk of malaria infection (confirmed by either a rapid diagnostic test or microscopy) as well as a reduced risk of moderate or severe anemia. These reductions were modest: approximately $10 \%$ for malaria infection and $5 \%$ for moderate or severe anemia. Importantly, however, the estimated pyrethroid resistance did not modify these reductions in any way. There was also agreement across both the pooled and meta-regression approaches used.

Selection bias was present in the data and occurred either because houses owning ITNs are predisposed to lower malaria transmission rates independent of ITN ownership or because ITN distribution programs are targeted to areas with higher malaria transmission; therefore, children in houses with ITNs are predisposed to more malaria transmission independent of ITN ownership. We attempted to mitigate the effects of this selection bias on the analysis using exact matching, as we and others have performed during previous analyses of ITN ownership using these data. ${ }^{9,35}$ It is possible that some residual bias is still present; if so, then we would expect that the bias would overinflate the effect of ITN ownership. However, if a decrease in ITN effectiveness occurred with increasing pyrethroid resistance, then we would still expect to see that effect because of the limitations of the data.

Modeled estimates of An. gambiae complex pyrethroid resistance with survey clusters were quite high (median, $90 \%$ ). Unfortunately, with such high levels of pyrethroid resistance spread across the continent, we could not examine the effectiveness of ITNs across the entire resistance range from $0 \%$ to $100 \%$. Because we included a large volume of data in our analyses, we still had sufficient power to determine that ITN ownership reduces the risk of malaria infection even when estimated pyrethroid resistance levels approach 100\% of the vector population.

These results are positive news for malaria control program. ITNs remain the primary vector control strategy throughout sub-Saharan Africa, and they appear to be effective even at high levels of pyrethroid resistance. Others have described this resistance paradox. During laboratory experiments, pyrethroid resistance conferred protection for malaria vectors; however, during epidemiological studies, vector control conferred protection against malaria infection. ${ }^{42}$ In general, there are two separate hypotheses regarding how ITNs maintain effectiveness in the presence of widespread resistance. The first hypothesis suggests that the same genes that confer pyrethroid resistance alter the ability of the vector to transmit the parasite. A fitness cost to pyrethroid resistance has been documented for An. gambiae, as evidenced by studies that have shown that nonresistant mosquitoes outcompete resistant mosquitoes. ${ }^{43}$ The second hypothesis suggests that the insecticide still functions, but with a different type of effect. One example of this second hypothesis is that pyrethroidresistant mosquitoes lose their irritancy to the insecticide, thus allowing for increased contact and a higher dose of the insecticide than that used for typical mosquitoes. ${ }^{44}$ This increased dose leads to the death of the mosquito. Another example of this second hypothesis is that a dose of pyrethroids results in delayed mortality of pyrethroid-resistant mosquitoes, ${ }^{45}$ perhaps because of the impaired flight or behavioral effects of the insecticide. ${ }^{46}$ Whatever the reason, ITNs still appear to effectively reduce malaria transmission, even when pyrethroid resistance approaches $100 \%$ in vector populations.
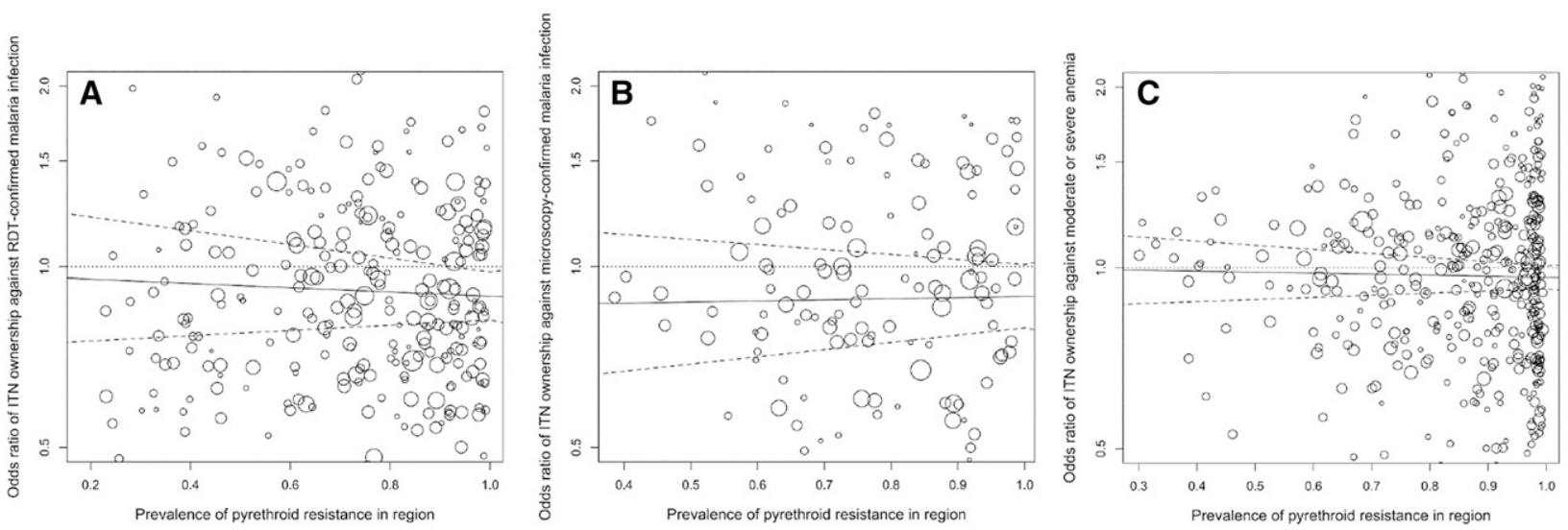

FIGURE 3. Bubble plots showing the relationship between pyrethroid resistance at the region level and effectiveness of insecticide-treated mosquito net (ITN) ownership against (A) malaria infection confirmed by a rapid diagnostic test (RDT), (B) microscopy-confirmed malaria infection, and (C) moderate or severe anemia. 
Received March 30, 2020. Accepted for publication May 20, 2021.

Published online August 23, 2021.

Financial support: RLC was supported by a grant from the Syracuse University Office of Undergraduate Research and Creative Engagement (SOURCE).

Authors' addresses: David A. Larsen and Rachael L. Church, Syracuse University Department of Public Health, Syracuse, NY, E-mails: dalarsen@syr.edu and rchurch@syr.edu.

\section{REFERENCES}

1. Eisele TP, Larsen DA, Walker N, Cibulskis RE, Yukich JO, Zikusooka CM, Steketee RW, 2012. Estimates of child deaths prevented from malaria prevention scale-up in Africa 2001-2010. Malar J 11: 93.

2. Murray CJL, Rosenfeld LC, Lim SS, Andrews KG, Foreman KJ, Haring D, Fullman N, Naghavi M, Lozano R, Lopez AD, 2012. Global malaria mortality between 1980 and 2010: a systematic analysis. Lancet 379: 413-431.

3. Bhatt $S$ et al., 2015. Coverage and system efficiencies of insecticide-treated nets in Africa from 2000 to 2017. eLife 4: e09672.

4. Bhatt S et al., 2015. The effect of malaria control on Plasmodium falciparum in Africa between 2000 and 2015. Nature 526: 207211.

5. World Health Organization, 2018. World Malaria Report. Geneva, Switzerland: WHO.

6. Rund SSC, O'Donnell AJ, Gentile JE, Reece SE, 2016. Daily rhythms in mosquitoes and their consequences for malaria transmission. Insects 7: 1-20.

7. Sutcliffe JF, Yin S, 2014. Behavioural responses of females of two anopheline mosquito species to human-occupied, insecticidetreated and untreated bed nets. Malar J 13: 294.

8. Lengeler C, 2004. Insecticide-treated bed nets and curtains for preventing malaria. Cochrane database of systematic reviews (Online): CD000363.

9. Larsen DA, Hutchinson P, Bennett A, Yukich J, Anglewicz P, Keating J, Eisele TP, 2014. Community coverage with insecticidetreated mosquito nets and observed associations with all-cause child mortality and malaria parasite infections. Am J Trop Med Hyg 91: 950-958.

10. Hawley WA et al., 2003. Community-wide effects of permethrintreated bed nets on child mortality and malaria morbidity in western Kenya. Am J Trop Med Hyg 68: 121-127.

11. Ranson H, Lissenden N, 2016. Insecticide resistance in African Anopheles mosquitoes: a worsening situation that needs urgent action to maintain malaria control. Trends in Parasitology 32: 187-196.

12. Cisse MBM, Sangare D, Oxborough RM, Dicko A, Dengela D, Sadou A, Mihigo J, George K, Norris L, Fornadel C, 2017. A village level cluster-randomized entomological evaluation of combination long-lasting insecticidal nets containing pyrethroid plus PBO synergist in southern Mali. Malar J 16: 477.

13. Gleave K, Lissenden N, Richardson M, Choi L, Ranson H, 2018. Piperonyl butoxide (PBO) combined with pyrethroids in insecticide-treated nets to prevent malaria in Africa. Cochrane Database Syst Rev 2018.

14. Tiono AB et al., 2018. Efficacy of Olyset Duo, a bednet containing pyriproxyfen and permethrin, versus a permethrin-only net against clinical malaria in an area with highly pyrethroidresistant vectors in rural Burkina Faso: a cluster-randomised controlled trial. Lancet 392: 569-580.

15. Hemingway J, Ranson $\mathrm{H}, 2000$. Insecticide resistance in insect vectors of human disease. Annu Rev Entomol 45: 371-391.

16. Santolamazza $F$ et al., 2008. Distribution of knock-down resistance mutations in Anopheles gambiae molecular forms in west and west-central Africa. Malar J 7: 1-8.

17. Ranson H, N'guessan R, Lines J, Moiroux N, Nkuni Z, Corbel V, 2011. Pyrethroid resistance in African anopheline mosquitoes: what are the implications for malaria control? Trends Parasitol 27: 91-98.
18. Hemingway $\mathrm{J}$ et al., 2016. Averting a malaria disaster: will insecticide resistance derail malaria control? Lancet 387: 1785-1788.

19. Kelly-Hope L, Ranson H, Hemingway J, 2008. Lessons from the past: managing insecticide resistance in malaria control and eradication programmes. Lancet Infect Dis 8: 387-389.

20. Thomas MB, Read AF, 2016. The threat (or not) of insecticide resistance for malaria control. Proc Natl Acad Sci USA 113: 8900-8902.

21. Opondo KO, Weetman D, Jawara M, Diatta M, Fofana A, Crombe F, Mwesigwa J, D'Alessandro U, Donnelly MJ, 2016. Does insecticide resistance contribute to heterogeneities in malaria transmission in the Gambia? Malar J 15: 1-10.

22. Mathanga DP, Mwandama DA, Bauleni A, Chisaka J, Shah MP, Landman KZ, Lindblade KA, Steinhardt LC, 2015. The effectiveness of long-lasting, insecticide-treated nets in a setting of pyrethroid resistance: a case-control study among febrile children 6 to 59 months of age in Machinga District, Malawi. Malar J 14: 1-8.

23. Kleinschmidt I et al., 2018. Implications of insecticide resistance for malaria vector control with long-lasting insecticidal nets: a WHO-coordinated, prospective, international, observational cohort study. Lancet Infect Dis 18: 640-649.

24. Tokponnon FT et al., 2019. Implications of insecticide resistance for malaria vector control with long-lasting insecticidal nets: evidence from health facility data from Benin. Malar $J$ 18: 1-9.

25. Bradley $\mathrm{J}$ et al., 2017. Insecticide-treated nets provide protection against malaria to children in an area of insecticide resistance in southern Benin. Malar J 16: 1-5.

26. Ochomo E et al., 2017. Insecticide-treated nets and protection against insecticide- resistant malaria vectors in Western Kenya. Emerg Infect Dis 23: 758-764.

27. Tusting LS, Bottomley C, Gibson H, Kleinschmidt I, Tatem AJ, Lindsay SW, Gething PW, 2017. Housing improvements and malaria risk in sub-Saharan Africa: a multi-country analysis of survey data. PLoS Med 14: 1-15.

28. Clarke SE et al., 2001. Do untreated bednets protect against malaria? Trans $R$ Soc Trop Med Hyg 95: 457-462.

29. Ochomo EO, Bayoh NM, Walker ED, Abongo BO, Ombok MO, Ouma C, Githeko AK, Vulule J, Yan G, Gimnig JE, 2013. The efficacy of long-lasting nets with declining physical integrity may be compromised in areas with high levels of pyrethroid resistance. Malar J 12: 1-10.

30. Hancock PA, Hendriks CJM, Tangena J-A, Gibson H, Hemingway J, Coleman M, Gething PW, Cameron E, Bhatt S, Moyes CL, 2020. Mapping trends in insecticide resistance phenotypes in African malaria vectors. PLoS Biol 18: e3000633.

31. Korenromp EL, Armstrong-Schellenberg JRM, Williams BG, Nahlen BL, Snow RW, 2004. Impact of malaria control on childhood anaemia in Africa - a quantitative review. Trop Med Int Health 9: 1050-1065.

32. Hijmans RJ, van Etten J, 2012. raster: Geographic Analysis and Modeling with Raster Data. Available at: http://cran.univlyon1.fr/web/packages/raster/.

33. R Core Development Team, 2010. R: a language and environment for statistical computing. Available at: http://wwwR-project. org/.

34. Moyes CL et al., 2019. Analysis-ready datasets for insecticide resistance phenotype and genotype frequency in African malaria vectors. Sci Data 6: 1-11.

35. Lim SS, Fullman N, Stokes A, Ravishankar N, Masiye F, Murray CJL, Gakidou E, 2011. Net benefits: a multicountry analysis of observational data examining associations between insecticide-treated mosquito nets and health outcomes. PLoS Med 8: e1001091.

36. Ho DE, Imai K, King G, Stuart EA, 2011. Matchlt: nonparametric preprocessing for parametric causal inference. J Stat Software 42: $1-28$.

37. Ho DE, Imai K, King G, Stuart EA, 2007. Matching as nonparametric preprocessing for reducing model dependence in parametric causal inference. Polit Anal 15: 199-236. 
38. Craig MH, Snow RW, le Sueur D, 1999. A climate-based distribution model of malaria transmission in sub-Saharan Africa. Parasitol Today 15: 105-111.

39. Gething PW, Patil AP, Smith DL, Guerra CA, Elyazar IRF, Johnston GL, Tatem AJ, Hay SI, 2011. A new world malaria map: Plasmodium falciparum endemicity in 2010. Malar $J$ 10: 378.

40. Sinka ME et al., 2011. The dominant Anopheles vectors of human malaria in Africa, Europe and the Middle East: occurrence data, distribution maps and bionomic précis. Parasit Vectors 4: 89.

41. Viechtbauer W, 2010. Conducting meta-analyses in R with the metafor package. J Stat Software 36: 1-48.

42. Alout H, Labbé P, Chandre F, Cohuet A, 2017. Malaria vector control still matters despite insecticide resistance. Trends Parasitol 33: $610-618$.
43. Lynd A, Weetman D, Barbosa S, Egyir Yawson A, Mitchell S, Pinto J, Hastings I, Donnelly MJ, 2010. Field, genetic, and modeling approaches show strong positive selection acting upon an insecticide resistance mutation in anopheles gambiae s.s. Mol Biol Evol 27: 1117-1125.

44. Chandre F, Darriet F, Duchon S, Finot L, Manguin S, Carnevale P, Guillet P, 2000. Modifications of pyrethroid effects associated with kdr mutation in Anophelos gambiae. Med Vet Entomol 14: 81-88.

45. Viana M, Hughes A, Matthiopoulos J, Ranson H, Ferguson HM, 2016. Delayed mortality effects cut the malaria transmission potential of insecticide-resistant mosquitoes. Proc Natl Acad Sci USA 113: 8975-8980.

46. Cohnstaedt LW, Allan SA, 2011. Effects of sublethal pyrethroid exposure on the host-seeking behavior of female mosquitoes. J Vector Ecol 36: 395-403. 